\title{
Gas vesicle protein expression for reconstitution of synthetic gas vesicles in yeast
}

\author{
Harin Jung ${ }^{1}$, Hua Ling ${ }^{1}$, Yong Quan Tan ${ }^{1}$, Nam-Hai Chua ${ }^{1}$, Wen Shan Yew ${ }^{1}$, and Matthew \\ Chang ${ }^{1}$
}

${ }^{1}$ National University of Singapore

February 7, 2021

\begin{abstract}
Given the potential applications of gas vesicles (GVs) in multiple fields including antigen-displaying and imaging, heterologous reconstitution of synthetic GVs is an attractive and interesting study that has translational potential. Here, we attempted to express and assemble GV proteins (GVPs) into GVs using the model eukaryotic organism Saccharomyces cerevisiae. We first selected and expressed two core structural proteins, GvpA and GvpC from cyanobacteria Anabaena flos-aquae and Planktothrix rubescens, respectively. We then optimized the protein expression conditions and validated GV assembly in the context of cell flotation and GV shapes. We found that when two copies of AnaA were integrated into the genome, it resulted in cell floatation and GV production regardless of GvpC expression. Next, we co-expressed chaperone-RFP with the GFP-AnaA to aid the AnaA aggregation. The co-expression of individual chaperones (Hsp42, Sis1, Hsp104, and GvpN) with AnaA led to the formation of larger inclusions and enhanced the sequestration of AnaA into the perivacuolar site. To our knowledge, this represents the first study on reconstitution of GVs in S. cerevisiae. Our results could provide insights into optimizing conditions for heterologous protein expressions as well as the reconstitution of other synthetic microcompartments in yeast.
\end{abstract}

\section{Hosted file}

Manuscript.pdf available at https://authorea.com/users/394236/articles/507664-gas-vesicleprotein-expression-for-reconstitution-of-synthetic-gas-vesicles-in-yeast

(a)

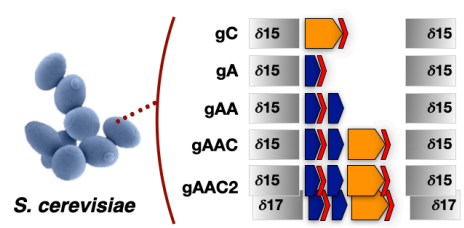

(b)

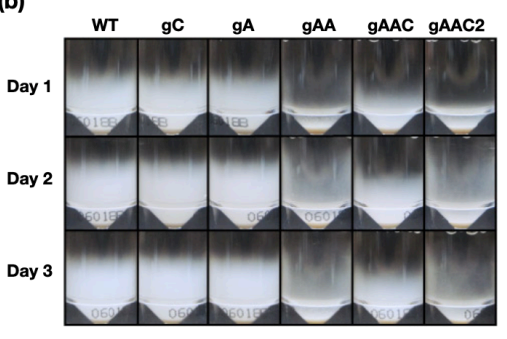

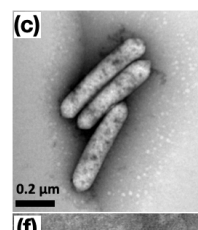
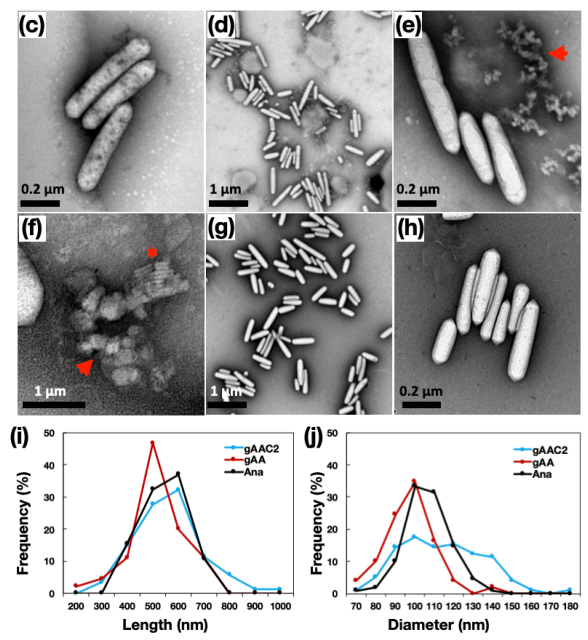
(a)

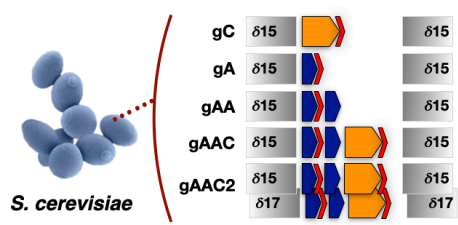

(b)

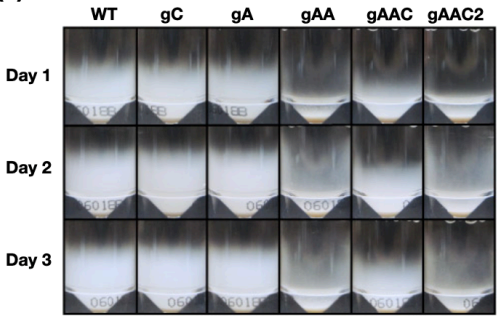

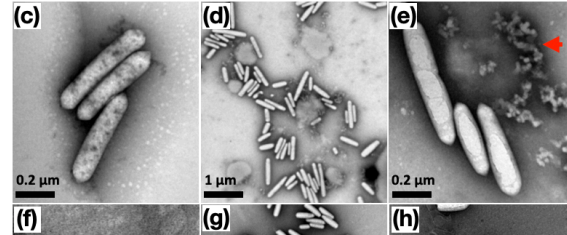
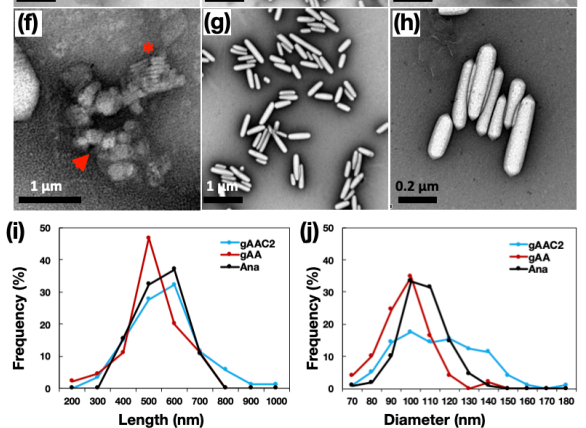

(a)

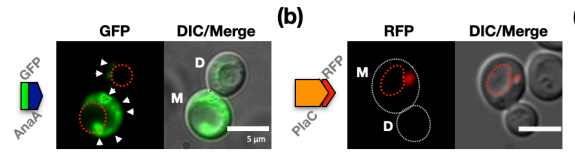

(c)

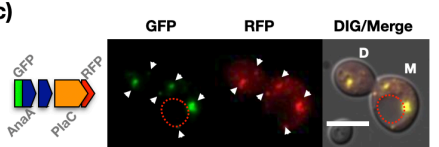

(d)

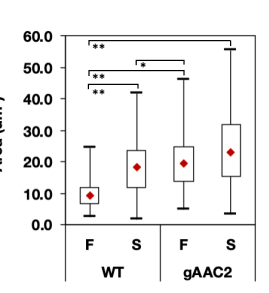

(e)
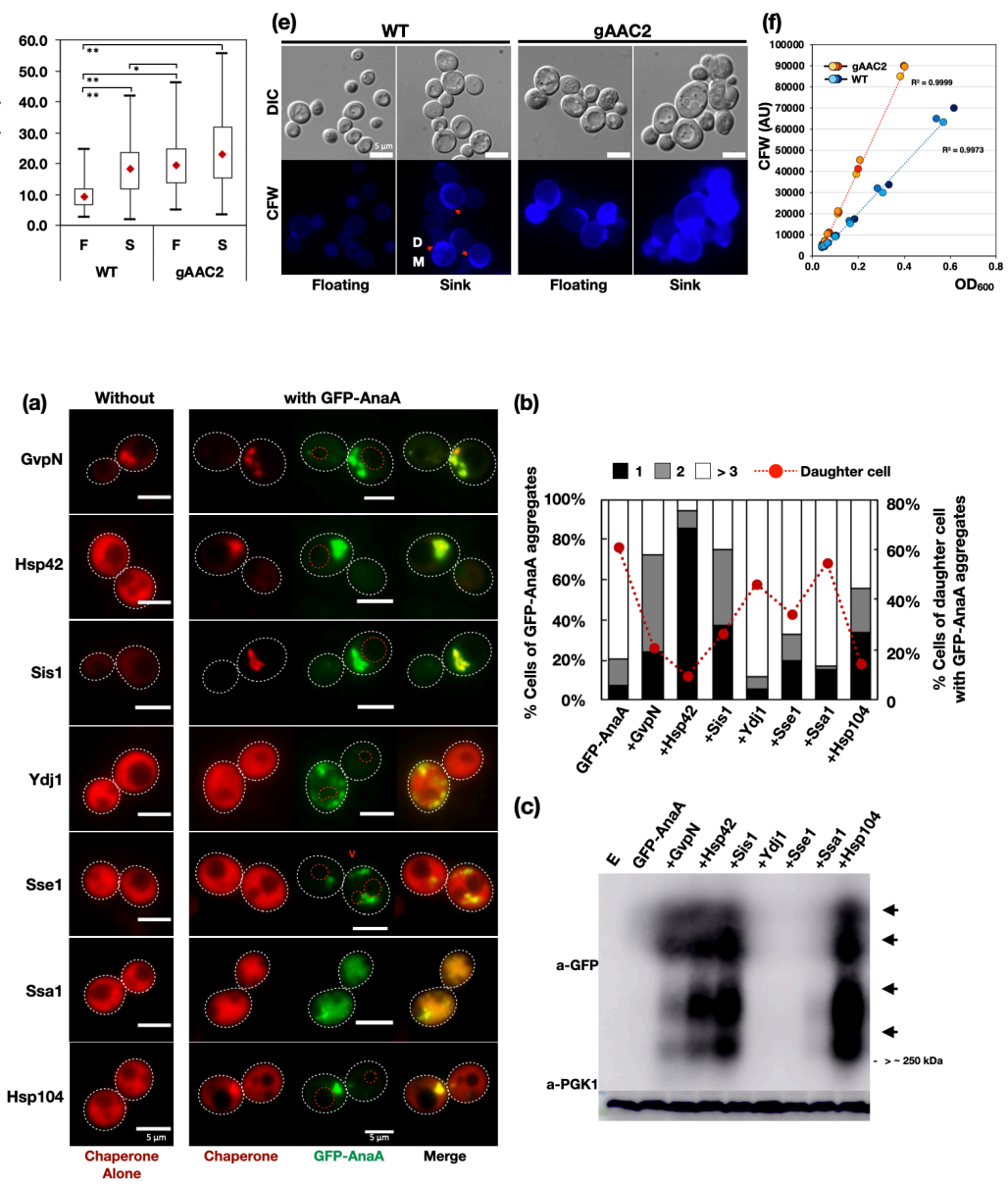

(b)

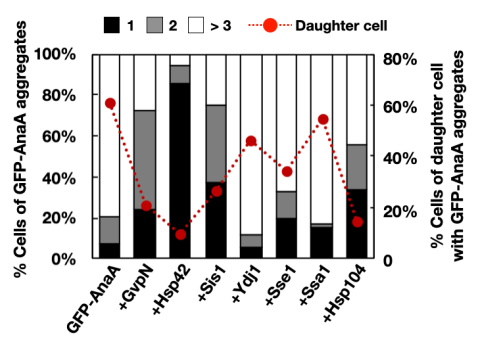

(c)

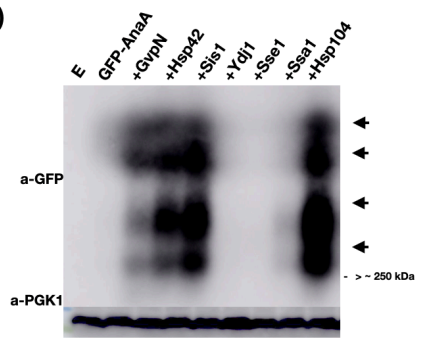

